\section{PM0-123 GENE TRANSFER OF DIMETHYLARGININE DIMETHYLAMINOHYDROLASE-1 REDUCES PORTAL PRESSURE IN A RODENT MODEL OF CIRRHOSIS}

\section{doi:10.1136/gutjnl-2012-302514b.123}

G Mehta, ${ }^{*}$ V Sharma, A Habtesion, V Balasubramaniyan, N A Davies, R Jalan, V Budhram-Mahadeo, R P Mookerjee. UCL, London, UK

Introduction Portal hypertensive bleeding is a grave complication of cirrhosis. Asymmetric dimethylarginine (ADMA), an endogenous eNOS inhibitor, is elevated in cirrhosis, relates to degree of portal hypertension, and is prognostic in acute-on-chronic liver failure. Dimethylarginine-dimethylaminohydrolase-1 (DDAH-1), a key enzyme metabolising hepatic ADMA, is reduced in cirrhosis. Therapies which indirectly increase hepatic DDAH-1, such as antiTNF $\alpha$ therapy or FXR agonists, lead to reduced hepatic ADMA, increased $\mathrm{NO}$ and lowered portal pressure. Therefore, there is accumulating evidence for DDAH-1 as a therapeutic target, but specific evidence for DDAH-1 reconstitution is lacking. The aim of this study was to adopt a DDAH-1 gene therapy strategy in portal hypertension.

Methods Hydrodynamic injection leads to hepatic gene transduction by causing turbulent, retrograde venous flow, permeation of hepatic parenchymal cells and consequent plasmid expression. Human DDAH-1 cDNA was cloned into the pCMV-Sport6 expression plasmid. Sprague-dawley rats $(n=9)$ underwent bile ductligation, and after 4 weeks were injected with $800 \mu \mathrm{g}$ of either pCMV-Sport6-DDAH1 $(n=5)$ or non-expressing control plasmid $(\mathrm{n}=4)$. After $72 \mathrm{~h}$, rats underwent direct portal pressure assessment under anaesthesia and were then sacrificed. Plasma ALT was measured by Cobas-Integra analyser. Transgene expression was measured by quantitative PCR, with Taqman probes specific for human DDAH-1 to distinguish rodent DDAH-1. Protein expression was measured by western blot.

Results Highly effective gene transfer (between 20 and 120-fold increase) of human DDAH-1 was seen in 3 out of 5 treated animals. None of the animals treated with control plasmid expressed human DDAH-1. The three "responders" to gene therapy also had highly significantly increased DDAH-1 protein expression compared with "non-responders" or controls $(\mathrm{p}<0.05)$. There was no difference in ALT between the groups. Portal pressure was significantly lower in "responders" to gene therapy than "non-responders" or animals treated with control plasmid ( $\mathrm{p}<0.05)$.

Conclusion This study demonstrates that DDAH-1 is a specific molecular target for portal pressure reduction. Hydrodynamic injection is variable in efficiency of gene delivery due to the nature of the technique. However, despite these limitations, this study clearly shows proof of concept for efficient vector-based DDAH-1 gene therapy in lowering portal pressure and preventing bleeding in cirrhosis.

Competing interests None declared.

\section{PM0-124 OXIDATIVE STRESS INDUCES ALTERNATIVE SPLICING AND NUCLEAR TRANSLOCATION OF KRUPPEL LIKE FACTOR 6}

doi:10.1136/gutjnl-2012-302514b.124

G Patman, ${ }^{*}$ K Y Hui, 0 M Anstee, H L Reeves. Northern Institute for Cancer Research, Newcastle University, Newcastle upon Tyne, UK

Introduction Progression of simple steatosis to NASH is attributed to inflammation and oxyradical overload ("oxidative stress"). Expression of the zinc finger transcription factor, KLF6, which up-regulates p21 and glucokinase, is increased in whole liver as disease progresses. KLF6 is regulated by alternative splicing and we propose that the balance of KLF6 alternative splice forms in different cellular compartments within the liver may influence the rate of NAFLD progression. We have therefore studied the impact of oxidative stress on KLF6 splice isoform expression.

Methods HepG2 cells were treated with either tertiary butyl hydrogen peroxide or angiotensin II, in the presence/absence of the anti-oxidant N-acteyl-L-cysteine (NAC). Reactive oxygen species were quantified by DCFDA and lucigenin chemiluminescence. KLF6 isoform expression and subcellular localisation was determined using variant specific real-time $\mathrm{PCR} /$ western blotting and immunofluorescence (IF).

Results While mRNA expression of both KLF6 full length (KLF6-FL) and its SV1/SV2 variants was markedly increased (10-50-fold) after exposure to oxidative stress, this occurred at $8 \mathrm{~h}$ and was unaffected by NAC. Expression of the little characterised KLF6-SV3 variant, however, was dramatically increased by over 30-fold at just $15 \mathrm{~min}$ Furthermore, this increase was significantly attenuated by $50 \%$ in the presence of NAC. Western blotting confirmed protein accumulation of both KLF6-FL and KLF6-SV3 at $30 \mathrm{~min}$, falling after $4 \mathrm{~h}$, with a sixfold increase in the KLF6 target gene, p21, at 4-8 h. KLF6 IF studies confirmed that both KLF6-FL and KLF6-SV3 in HepG2 cells translocate to the nucleus after exposure to oxidative stress. In human NAFLD, a modest 1.5-2-fold increase in KLF6-FL mRNA contrasted sharply with a dramatic ninefold increase in KLF6-SV3 mRNA $(p<0.01)$ in tissues with inflammation $(n=16)$ compared to those without $(n=7)$.

Conclusion While KLF6-FL clearly accumulates in the nucleus of HepG2 cells in response to oxidative stress, it is the little characterised KLF6-SV3 isoform which is redox sensitive at the level of transcription and which is dramatically increased in association with inflammation in NAFLD. This variant retains the NLS and the first zinc finger of the DNA binding domain. Further characterisation of its functions and targets will help us to understand its role in NAFLD progression.

Competing interests None declared.

\section{PM0-125 NEUTROPHIL INTRACELLULAR TOLL-LIKE RECEPTOR (TLR) 9 EXPRESSION SERVES AS A BIOMARKER THAT DETERMINES PRESENCE AND SEVERITY OF ENCEPHALOPATHY IN ACUTE LIVER FAILURE AND CIRRHOSIS}

doi:10.1136/gutjnl-2012-302514b.125

${ }^{1} \mathrm{G}$ K Manakkat Vijay, ${ }^{1} \mathrm{R}$ D Abeles, ${ }^{1} \mathrm{~S}$ Ramage, ${ }^{2} \mathrm{~A}$ Riva, ${ }^{1} \mathrm{~J} \mathrm{M}$ Ryan, ${ }^{1} \mathrm{~N}$ J Taylor, 1J A Wendon, ${ }^{2} \mathrm{~S}$ Chokshi, ${ }^{1} \mathrm{Y}$ Ma, ${ }^{1} \mathrm{D}$ L Shawcross. Institute of Liver Studies and Transplantation, King's College London School of Medicine at King's College Hospital, Denmark Hill, London, UK; ${ }^{2}$ Foundation for Liver Research, London, UK

Introduction There is a marked propensity for patients with acute liver failure (ALF) and cirrhosis to develop sepsis and inflammation which may hasten the development of hepatic encephalopathy (HE) and cerebral oedema. Neutrophil dysfunction is an important biomarker of poor prognosis in liver failure and neutrophil TLR9 expression upregulates with ammonia exposure. There is a paucity of understanding regarding the relationship between neutrophil dysfunction and the development of HE, moreover there is a lack of predictive/prognostic biomarkers that differentiate ALF patients that will go on to develop HE. The aim of this study was to investigate the relationship between neutrophil TLR9 and development of $\mathrm{HE}$

Methods In healthy controls $(n=12)$ and patients with ALF $(n=12)$ and cirrhosis $(n=50)$ we investigated neutrophil TLR9 expression using fluorochrome-conjugated monoclonal antibodies [CD16 (PE), CD11b (APC-Cy7) and TLR9 (APC) (after cytofixation/permeabilisation)] by flow cytometry in determining responses to endotoxin 